\title{
Natural Image Enhancement Using a Biogeography Based Optimization Enhanced with Blended Migration Operator
}

\author{
J. Jasper, ${ }^{1}$ S. Berlin Shaheema, ${ }^{2}$ and S. Berlin Shiny ${ }^{3}$ \\ ${ }^{1}$ Department of Electrical Engineering, Anna University, Regional Centre, Coimbatore, Tamil Nadu 641047, India \\ ${ }^{2}$ Department of Computer Science and Engineering, Jayamatha Engineering College, Aralvaimozhi, Tamil Nadu 629301, India \\ ${ }^{3}$ Department of Computer Science and Engineering, Ponjesly College of Engineering, Nagercoil, Tamil Nadu 629003, India
}

Correspondence should be addressed to J. Jasper; mailtojasper@gmail.com

Received 23 November 2013; Accepted 6 February 2014; Published 17 March 2014

Academic Editor: Anand Paul

Copyright (C) 2014 J. Jasper et al. This is an open access article distributed under the Creative Commons Attribution License, which permits unrestricted use, distribution, and reproduction in any medium, provided the original work is properly cited.

\begin{abstract}
This paper addresses a novel and efficient algorithm for solving optimization problem in image processing applications. Image enhancement (IE) is one of the complex optimization problems in image processing. The main goal of this paper is to enhance color images such that the eminence of the image is more suitable than the original image from the perceptual viewpoint of human. Traditional methods require prior knowledge of the image to be enhanced, whereas the aim of the proposed biogeography based optimization (BBO) enhanced with blended migration operator (BMO) algorithm is to maximize the objective function in order to enhance the image contrast by maximizing the parameters like edge intensity, edge information, and entropy. Experimental results are compared with the current state-of-the-art approaches and indicate the superiority of the proposed technique in terms of subjective and objective evaluation.
\end{abstract}

\section{Introduction}

Image enhancement plays a vital role in a variety of fields such as vision, medical image analysis, remote sensing, and in our real life photographic images [1]. Since images can worsen due to poor quality of the acquisition device, lack of proficiency of the operation, and the climatic conditions at the time of acquisition. Such image may not expose all the fine points in the captured scene and may have a dull and aberrant look. Hence, it has become a necessity to develop algorithms for image enhancement. The image enhancement (IE) approach improves the quality of the image by extracting critical details of the images to improve the interpretability or perception of the image for human viewers. IE accentuates or sharpens image features such as edge boundaries or contrast to make a graphic display more beneficial to further image applications such as enhancing edges and image segmentation and to enforce the capability of machine recognition systems to interpret useful information in the image [2]. It has become a necessity to develop tools and algorithms for color image enhancement with the rapid increase in the usage of color images.

Histogram transformation is considered one of the fundamental processes for image enhancement of gray level images [3], which facilitates subsequent higher level operations such as detection and identification. Histogram equalization [4] and contrast manipulations [5] are well-known methods for enhancing the contrast of a given image but most of them tend to be heuristic based on deep expert knowledge for image processing. Hence these techniques require a large amount of analysis and computation because of complicated formulations. Histogram modification framework reveals maximum information contained in the image indirectly modifies the image histogram [6]. weighted threshold HE (WTHE), [7-11] suggests modifying the image histogram by weighting and threshold before histogram equalization. These methods modify the image histogram indirectly and reveal the maximum information contained in the image.

Optimization plays an imperative role in computer science, artificial intelligence, operational research, and other 
related fields $[12,13]$. It is the process of trying to find the best possible solution to an optimization problem within a reasonable time limit. Several evolutionary algorithms (EA) such as genetic algorithm (GA), particle swarm optimization (PSO), and differential evolution (DE) have been introduced in recent years in the field of image processing because of their fast computing ability. Several meta heuristics method mainly EA, PSO,GA, DE etc., have been applied for image processing applications [12-15] including image enhancement problem. GA finds weights and combines four types of nonlinear transform elements [16]. In [17] a real coded GA to adapt the gray level intensity transformation in the image with an evaluation criterion [18] combines different transformation functions with different parameters to produce enhanced image. Genetic algorithm (GA) [19-21] is used to minimize entropy value in order to enhance the image and to preserve the information. DE is applied to solve image enhancement for gray scale images [22]. PSO based image enhancement [23-27] is introduced; [28] cuckoo search algorithm [18, 29], a fuzzy logic approach to image enhancement, takes less time to converge to a better optimal. It gives poor contrast without proper tuning of parameters.

Generalizing gray scale image enhancement to color image enhancement is not a trivial task. Several factors must be considered for color image enhancement such as selection of a color model, characteristics of the human visual system, and color contrast sensitivity. Multiscale approach to contrast enhancement [2] uses a nonlinear scale-space representation of image generated by anisotropic diffusion. Another multiscale contrast enhancement technique through nonlinear pyramid recombination is introduced in [30, 31]. Color images can also be enhanced by separating the image into chromaticity and intensity components [11]. In [32] color enhancement based on saturation is proposed. A method for color equalization with its application to color images is proposed in [33]. A space-variant luminance map based color image enhancement is proposed in [34]. A scheme for color image enhancement employing genetic algorithm is proposed in [17]. A hue preserving and gamut problem free color image enhancement is proposed in [35] considering RGB and CMY color spaces. In $[36,37]$ color image enhancement using Artificial Bee Colony is proposed.

In this paper the color image enhancement is approached as a constrained optimization problem. In comparison to other optimization algorithms the solutions do not die at the end of each generation [38]. At the same time BBOBMO does not take unnecessary computational time. Our objective is to maximize the objective function in order to enhance the contrast by maximizing the number of pixels in the edges, increase the overall edges intensity, and increase the entropy measure. The color contrast of the image is preserved by adjusting the background illumination and prevents the degradation of the image pixels. The resulted color enhanced images by BBO-BMO are found to be better compared with other image enhancement techniques. Both objective and subjective evaluations on the resulted image show the goodness of BBO-BMO.

The rest of the paper is organized as follows. Section 2 presents the fundamentals of image enhancement. In
Section 3 the concepts BBO and BMO are described. Implementation of the proposed biogeography based optimization and blended migration operation (BBO-BMO) for image enhancement algorithm is described in Section 4. Experimental results are presented in Section 5 to describe the advantage of color image enhancement using our algorithm. Finally conclusions are addressed in Section 6.

\section{Image Enhancement}

Image enhancement improves the visual appearance of an image by increasing its contrast and sharpening the features. Image enhancement technique requires a transformation function which takes the intensity value of each pixel from the input image and generates a new intensity value to produce the enhanced image. The quality of the enhanced image is evaluated by the evaluation function.

2.1. Enhancement Function. Image enhancement done on spatial domain generates a new intensity value for each pixel of the original image to generate the enhanced image. The enhancement process can be denoted by

$$
g(i, j)=T(f(i, j)),
$$

where $f(i, j)$ is the gray value of the input image $I(i, j)$ and $g(i, j)$ is the enhanced image; $T$ is the transformation function. The transformation $T$ is defined as

$$
\begin{aligned}
g(i, j)= & K(i, j)[f(i, j)-c \times m(i, j)] \\
& +m(i, j)^{a}
\end{aligned}
$$

where $a$ and $c$ are two parameters whose value is to be optimized, $m(i, j)$ is the local mean of the $(i, j)$ th pixel of the input image over an $n \times n$ window, and $K(i, j)$ is the enhancement function which takes both global and local information.

The local mean is given as

$$
m(i, j)=\frac{1}{n \times n} \sum_{x=0}^{n-1} \sum_{y=0}^{n-1} f(x, y) .
$$

The enhancement function $K(i, j)$ is defined as

$$
K(i, j)=\frac{k \cdot D}{\sigma(i, j)+b},
$$

where $k$ and $b$ are the two parameters to be optimized, $\sigma(i, j)$ is the local standard deviation of input image, and $D$ is the global mean.

The global mean is defined as

$$
D=\frac{1}{M \times N} \sum_{x=0}^{M-1} \sum_{y=0}^{N-1} f(x, y),
$$

where $M$ and $N$ are the number of pixels in the horizontal and vertical direction of the image. The local standard deviation $\sigma(i, j)$ is defined as

$$
\sigma(i, j)=\sqrt{\frac{1}{n \times n} \sum_{x=0}^{n} \sum_{y=0}^{n}(f(x, y)-m(i, j))^{2}} .
$$


The transformation function is defined as

$$
g(i, j)=\left[\frac{k \cdot D}{\sigma(i, j)+b}[f(i, j)-c \times m(i, j)]+m(i, j)^{a}\right] \text {. }
$$

By this transformation equation the contrast of the image is enhanced considering local mean to be the center of enhancement. The term $m(i, j)^{a}$ has brightening and smoothing effect and thus smoothes the enhanced image and the four parameters introduced in the transformation function; that is, $a, b, c$ and $k$ are the parameters of the enhancement function and the small variation in their value produces a large variation in the processed image and thus the value of these parameters should be precisely set. The approximate range of these parameters is defined [39] as $a[0,1.5], b[0,(D / 2)], c[0,1]$, and $K[0.5,1.5]$.

2.2. Enhancement Evaluation Criterion. To evaluate the quality of an enhanced image without human intervention needs an objective function. Many objective functions are presented in the literature [40-42]. The objective function is obtained combining the performance measures entropy value, sum of the edge intensities, and edge pixels and is defined as

$$
F(Z)=\frac{\log (\log (E(I(Z)))) \cdot n e((I(Z))) \cdot H(I(Z))}{M \times N},
$$

where $F(Z)$ is the fitness function applied to species $Z$. $I(Z)$ denotes original image $I$ with a transformation $T$ applied according to (7) where the respective parameters $a, b, c$, and $k$ are given by the species $Z=(a, b, c, k)$.

$E\left(I_{s}(Z)\right)$ is the intensity of the edges detected with edge detector, where the detector is applied to the transformed image $I(Z)$. ne is the number of edge pixels detected with the edge detector.

$H(I(Z))$ is the entropy of the image $I(Z)[26]$ :

$$
H(I(Z))=-\sum_{i=0}^{255} e_{i}
$$

where $e_{i}=h_{i} \log _{2}\left(h_{i}\right)$ if $h_{i} \neq 0$; otherwise $e_{i}=0$ and $h_{i}$ is the probability of occurrence of $i$ th intensity value of enhanced image.

\section{Theory of BBO-BMO: An Overview}

3.1. Biogeography Based Optimization. Biogeography based optimization is a new population based optimization algorithm proposed by Simon [38] in 2008. BBO is based on the study of the distribution of species and is characterized by two probabilistic operators, that is, migration and mutation. The species are distributed in habitats that are geographically isolated from other habitats. The suitability of residing in a habitat is determined by a dependent variable known as habitat suitability index (HSI). The variables that characterize habitability are called suitability index variables (SIVs). Habitats with high HSI have large number of species while habitats with low HSI have small number of species. Migration refers to the movement of species from one habitat to another habitat. This movement of species is always from habitat with high HSI to habitat with low HSI.

Sometimes due to sudden changes in natural calamities or other events the HSI of a habitat might get changed. This event of sudden change in HSI of a habitat is represented as mutation of SIV in BBO. Mutation in each habitat is controlled by its species count probability.

3.2. Blended Migration Operator. Migration refers to the flow of species between any two habitats. Habitats with high HSI have low immigration rate and high emigration rate, while habitats with low HSI have low immigration rate and high emigration rate. Thus high HSI habitats are more static in species distribution while low HSI habitats are dynamic. The concept of migration is expressed as

$$
H_{i}(\mathrm{SIV}) \longleftarrow H_{j}(\mathrm{SIV}) .
$$

Equation (10) shows that the species from habitat $H_{j}$ is emigrating to the habitat $H_{i}$. The probability that $H_{j}$ is selected as the emigrating habitat is proportional to the emigration rate $\mu_{j}$ and the probability $H_{i}$ selected as immigrating habitat is proportional to its immigration rate $\lambda_{i}$. A good solution has high $\mu$ and low $\lambda$ and vice versa for poor solution. The immigration and emigration rate of a habitat is calculated as below:

$$
\begin{aligned}
\lambda_{i} & =I\left(1-\frac{k(i)}{n}\right), \\
\mu_{i} & =E^{*}\left(\frac{k(i)}{n}\right),
\end{aligned}
$$

where $I$ and $E$ are maximum immigration and emigration rates, respectively. $k(i)$ is the fitness rank of the $i$ th individual and $n$ is the number of candidate solutions in a population.

In this work, a new migration operator is presented called as blended migration operator (BMO) [43]. The concept of blended migration is borrowed from the blended crossover in GA. Unlike the standard $\mathrm{BBO}$, in blended migration a solution feature of $H_{i}$ is not simply replaced by a new solution feature from $H_{j}$. Instead, a new solution feature of $H_{i}$ is comprised of two migrated components: first, the solution feature from a habitat $H_{j}$ and, second, the solution feature from itself. The blended migration is expressed as

$$
H_{i}(\mathrm{SIV}) \longleftarrow \alpha H_{i}(\mathrm{SIV})+(1-\alpha) H_{j}(\mathrm{SIV}),
$$

Equation (13) shows that the new solution feature of $H_{i}$ comes from a combination of its own SIV and emigrating habitat $H_{j}$. Blended migration is more advantageous than the normal migration. Due to blended migration good solutions are less likely to be degraded and poor solutions can still accept a lot of new features from the good solutions.

3.2.1. Mutation. Random events such as disease and natural catastrophes, cause the species count of a habitat to differ from its equilibrium value. Mutation in $\mathrm{BBO}$ refers to the random modification of a habitat's SIV based on its mutation 


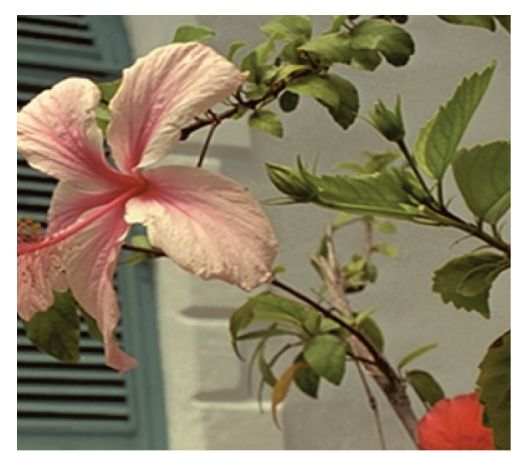

(a)

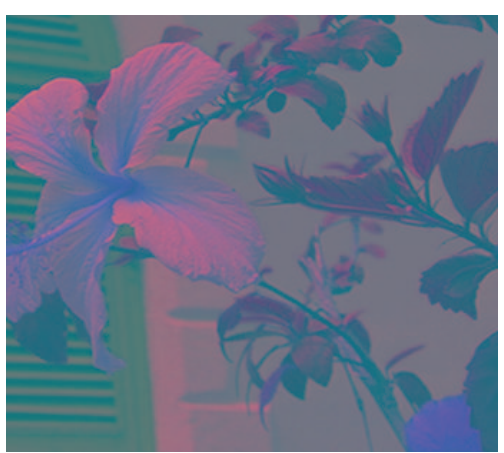

(b)

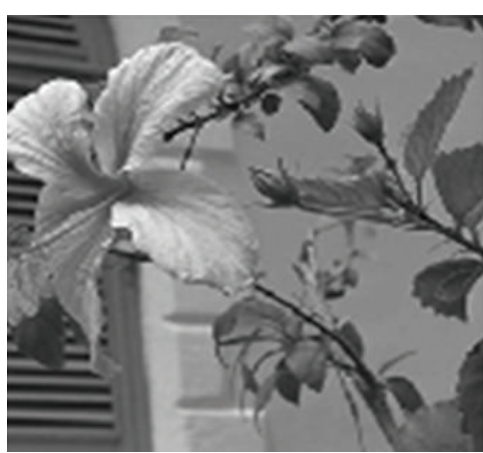

(c)

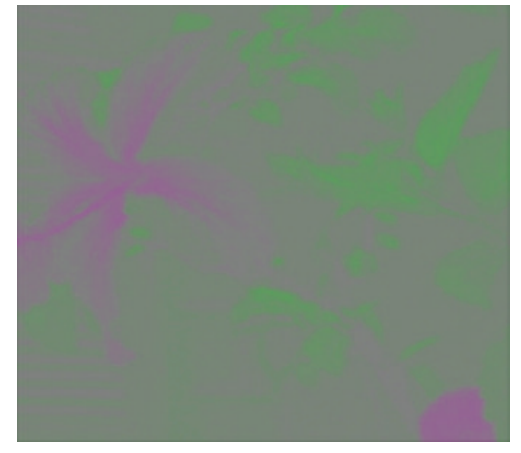

(d)

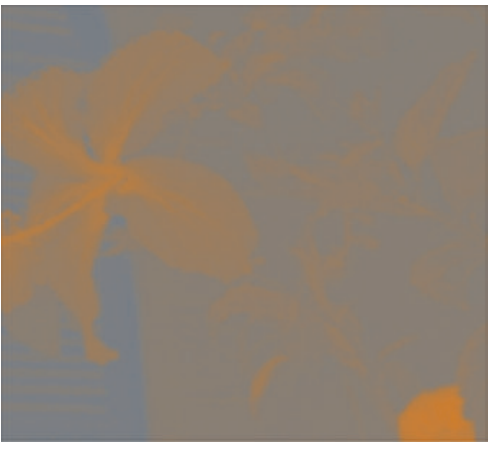

(e)

FIGURE 1: Color conversion obtained of the natural images: (a) original image, (b) CIEL ${ }^{*} a^{*} b^{*}$ color model, (c) lightness image, (d) $a^{*}$ coloropponent dimensions image, and (e) $b^{*}$ color-opponent dimensions image.

probability. For standard $\mathrm{BBO}$, the mutation probability is inversely proportional to the solution probability and it is expressed as

$$
m_{i}=m_{\max }\left(1-\frac{P_{i}}{P_{\max }}\right),
$$

where $m_{\max }$ is the maximum mutation probability and $P_{i}$ is the species count probability. The probability of species counts for each habitat is governed by a differential equation given as

$$
\begin{aligned}
& \dot{P}_{s} \\
& \quad \begin{cases}-\left(\lambda_{s}+\mu_{s}\right) P_{s}+\mu_{s+1} P_{s+1}, & S=0 \\
-\left(\lambda_{s}+\mu_{s}\right) P_{s}+\mu_{s+1} P_{s+1}+\lambda_{s-1} P_{s-1}, & 1 \leq S \leq S_{\max }-1 \\
-\left(\lambda_{s}+\mu_{s}\right) P_{s}+\lambda_{s-1} P_{s-1}, & S=S_{\max },\end{cases}
\end{aligned}
$$

where $\lambda_{s}$ and $\mu_{s}$ are immigration and emigration rates of a habitat when there are $S$ species. Mutation is carried on both good solutions and poor solutions. It helps improve the low HSI solution and provides high HSI solution improving even more than they already have.

\section{Implementation of BBO-BMO for Image Enhancement}

The Image enhancement using BBO-BMO algorithm can be described with the following algorithm.
Step 1 (image preprocessing). (a) Color Conversion. Obtain the color image and convert it into CIEL ${ }^{*} a^{*} b^{*}$ color model. Image enhancement of color images is typically done by transforming an image to a color space that has image intensity as one of its components. CIEL ${ }^{*} a^{*} b^{*}$ color spaces are used as a part of the color image pipeline in video and digital photography systems. Manipulating luminosity affects the intensity of the pixels while preserving the original colors. Figure 1 illustrates the color conversion model obtained in the natural image.

(b) Noise Separation. Noise reduction [44, 45] should attempt to remove noise without sacrificing real detail from the scene photographed. Color images find chroma noise more objectionable than luminance noise; the colored blobs are considered "digital looking" and unnatural, compared to the grainy appearance of luminance noise that some compare to film grain. Adaptive bilateral filter attempts to remove noise without sacrificing real detail from the natural image; one risks a greater loss of detail from luminance noise reduction than chroma noise reduction because most scenes have high frequency chroma detail. For these reasons, our noise reduction filter splits the image detail into chroma and luminance components and applies more noise reduction by preserving the sharpness.

(c) Edge Detection. Apply edge detector to detect the edges by combining the filter which increases the edge slope of the 


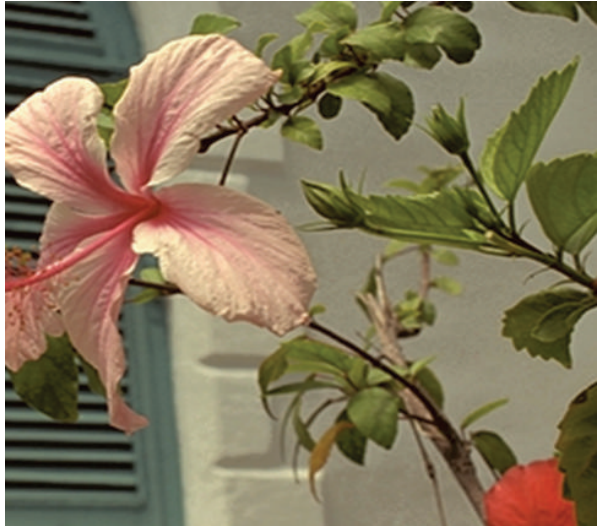

(a)

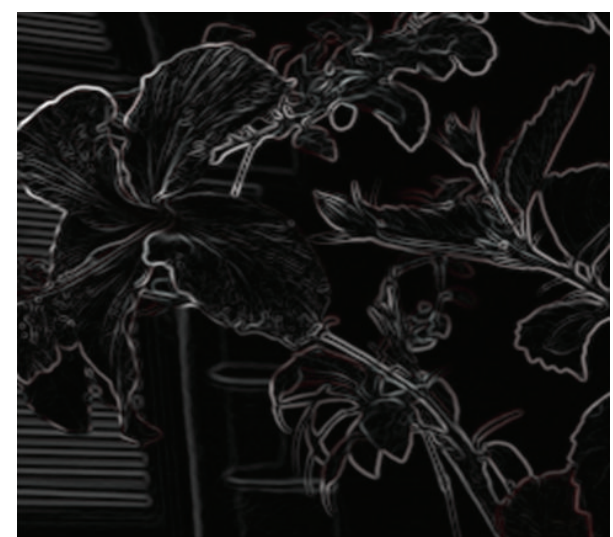

(b)

FIgURE 2: Illustrations of edge detection of the natural images: (a) original image and (b) edge detection.

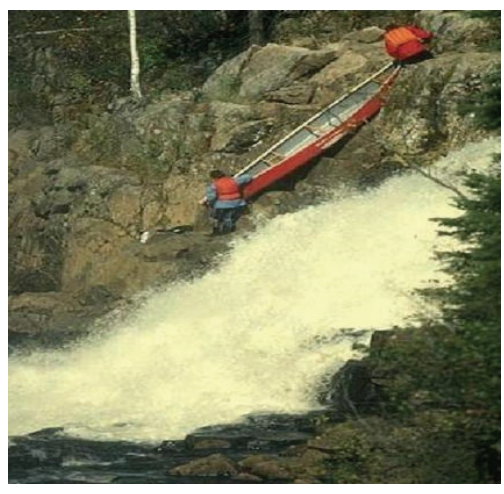

(a)

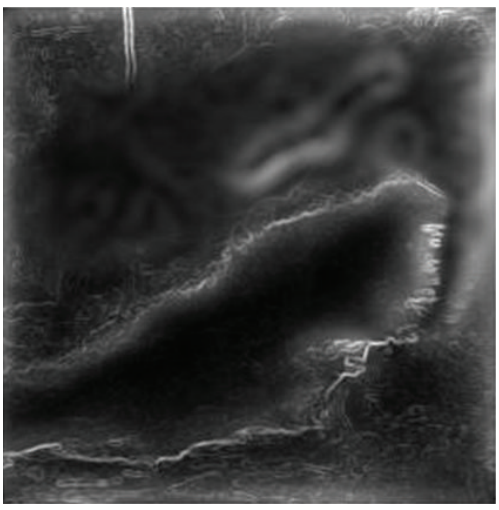

(d)

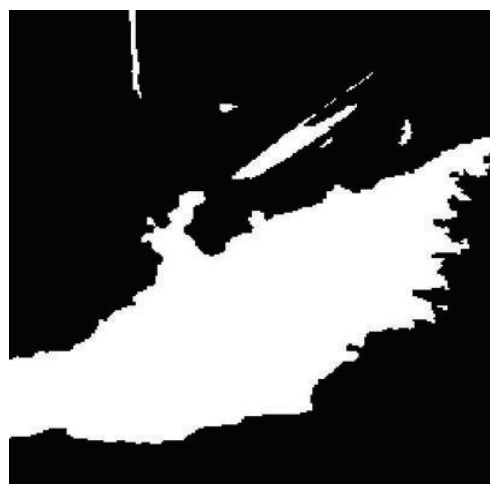

(b)

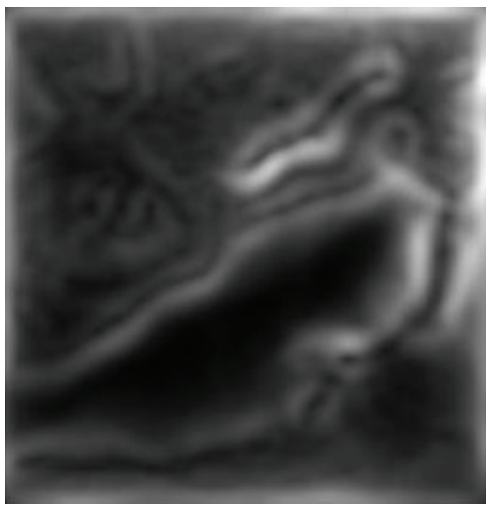

(e)

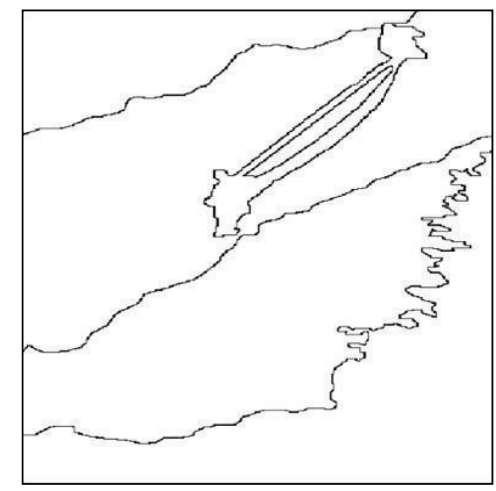

(c)

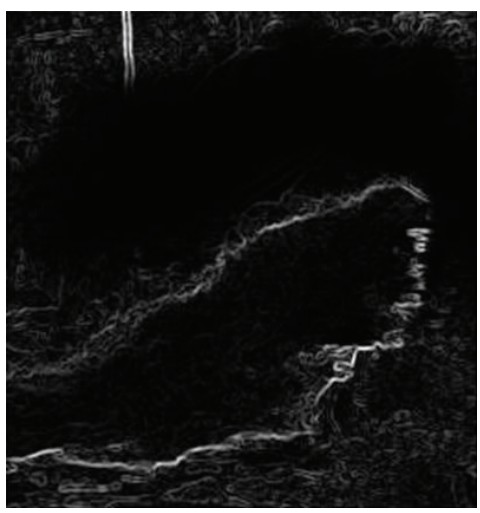

(f)

Figure 3: Illustrates texture and edge intensity of the Natural images: (a) Original image (b) texture image (c) edge detection (d) texture gradient (e) gradient map (f) edge entropy.

given image for obtaining proper edge enhanced image [46]. Figure 2 illustrates the edge detection process.

Step 2 (initialization). Initialize the BBO parameter such as number of habitats $N$ is the total number of image pixels, habitat modification probability $P_{\text {mod }}$, mutation probability $P_{\text {mut }}$, maximum mutation rate $m_{\max }$, maximum immigration rate $I$, maximum emigration rate $E$, lower limit for immigration probability per gene $\lambda_{\text {lower }}$, upper limit for immigration probability per gene $\lambda_{\text {upper }}$, step size for numerical integration $d t$, elitism parameter $p$, and maximum number of iteration. Also initialize the maximum and minimum pixel value of an image, that is, number of SIV $m$. Since the real comparison of pixel values is the decision variables for the problem, they 


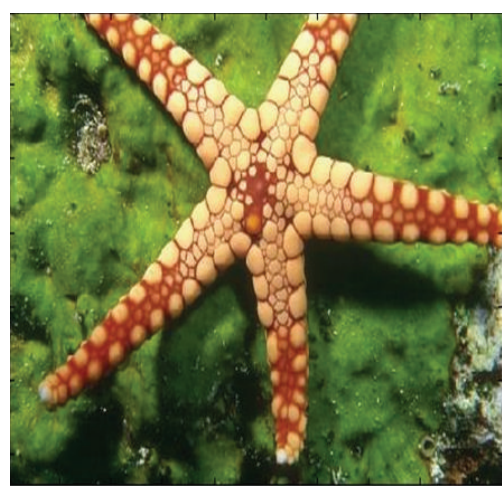

(a)

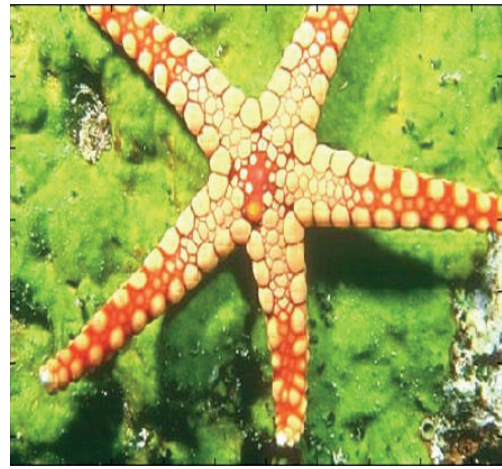

(d)

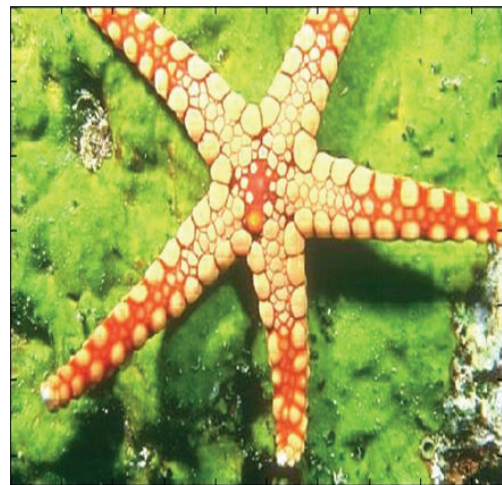

(g)

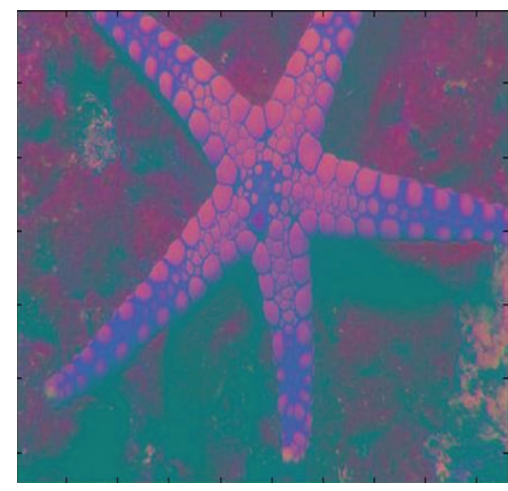

(b)

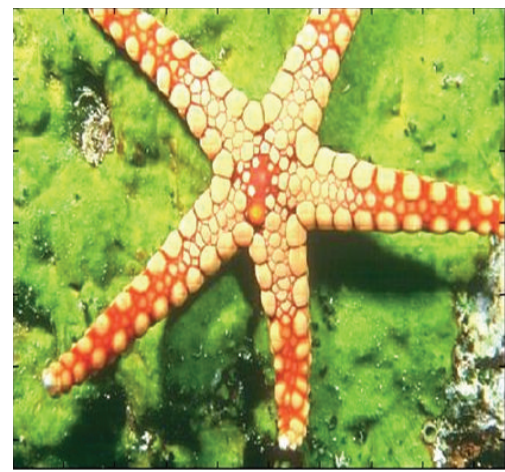

(e)

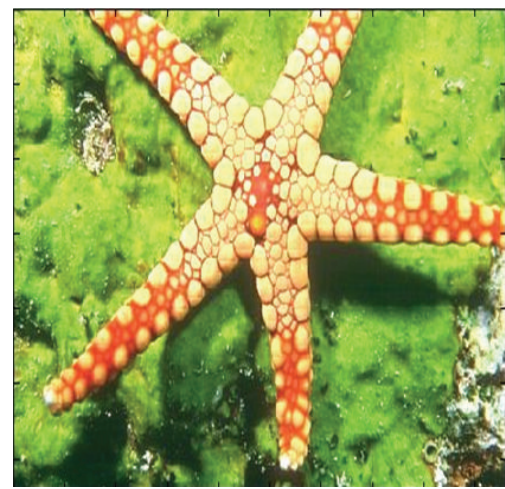

(h)

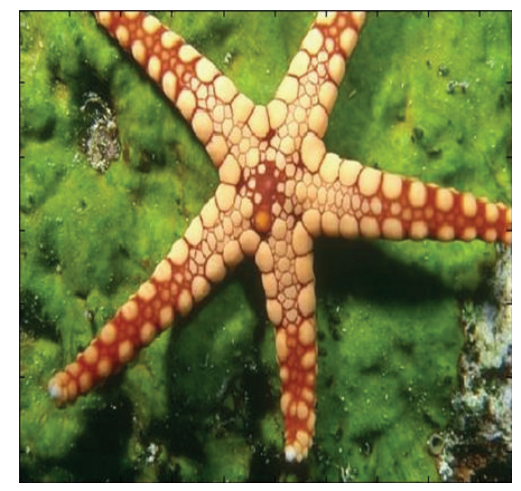

(c)

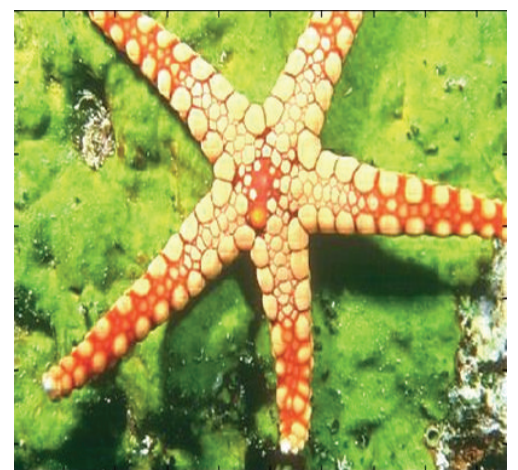

(f)

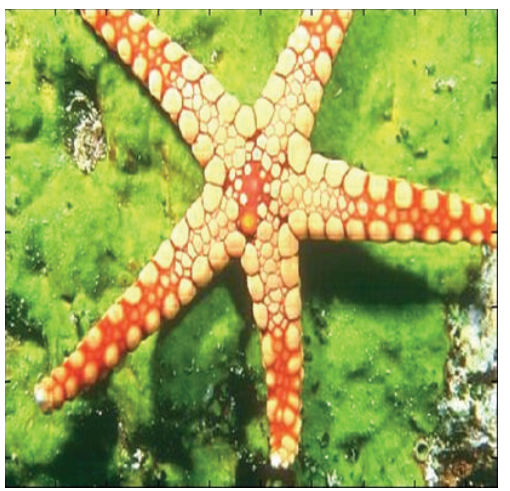

(i)

Figure 4: Typical results obtained from the compared algorithms of the natural image: (a) original image, (b) CIEL ${ }^{*} a^{*} b^{*}$ color model, (c) noisy and blurred image, (d) WTHE, (e) fuzzy, (f) GA, (g) PSO method, (h) DE method, and (i) proposed method.

are represented as SIV in a habitat. A population of habitat is represented as

$$
H=\left[H^{1}, H^{2}, \ldots H^{i}, \ldots H^{N}\right] .
$$

Each habitat consists of $m$ number of SIVs. An individual habitat $H_{i}$ with SIVs is represented as

$$
\begin{aligned}
H^{i} & =\operatorname{SIV}^{i j}=\left[\operatorname{SIV}^{i 1}, \operatorname{SIV}^{i 2}, \ldots, \operatorname{SIV}^{i m}\right] \\
& =\left[P^{i 1}, P^{i 2}, \ldots, P^{i m}\right],
\end{aligned}
$$

where $i=1,2, \ldots, N$ and $j=1,2,3, \ldots, m$. Each habitat is one of the possible solutions for the problem. In a habitat $H^{i}$ the component $S V^{i j}$ refers to the pixel value of the $m$ th pixel of the $i$ th habitat.

Step 3 (initialization of SIV). Each SIV in the matrix $H$ is randomly initialized within the minimum and maximum pixel value of the neighborhood pixels, respectively.

Step 4 (evaluation of fitness of the population). Calculate the fitness of each habitat which is known as HSI in BBO. The fitness is calculated using a function $f$ given in (8). If the fitness of previous pixel value is greater than the current pixel value after considering fitness evaluation which depends upon global intensity value of image, it should be replaced. 


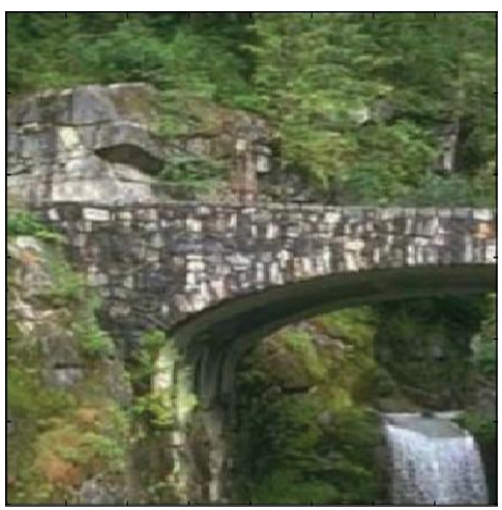

(a)

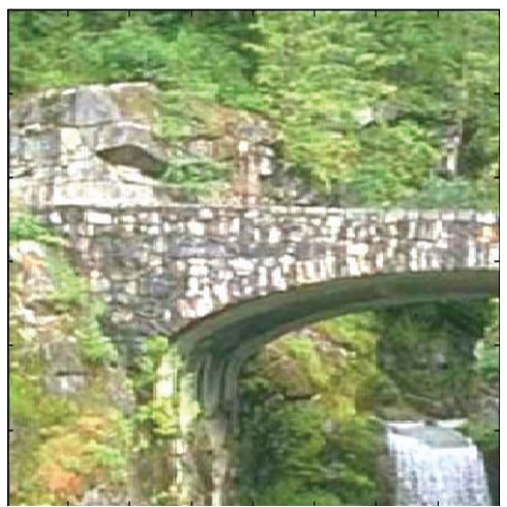

(d)

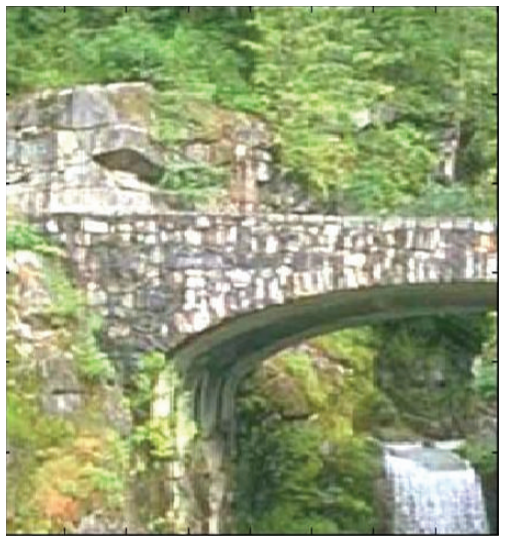

(g)

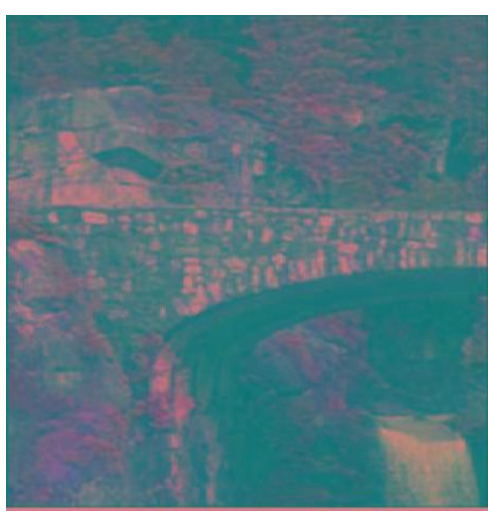

(b)

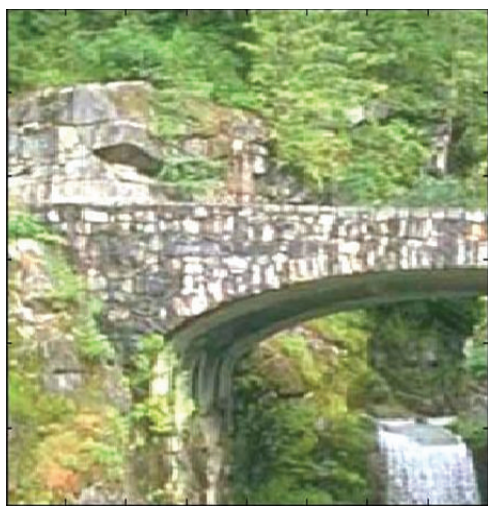

(e)

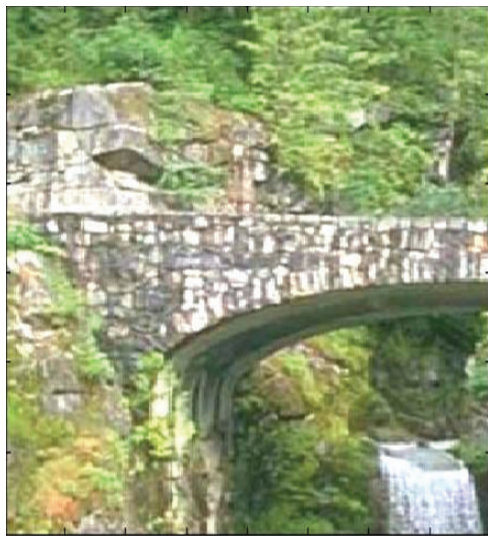

(h)

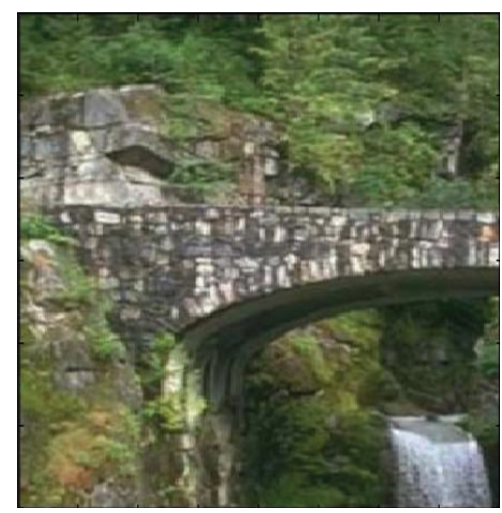

(c)

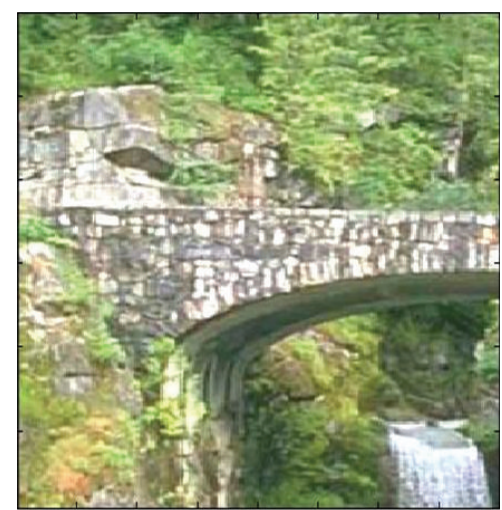

(f)

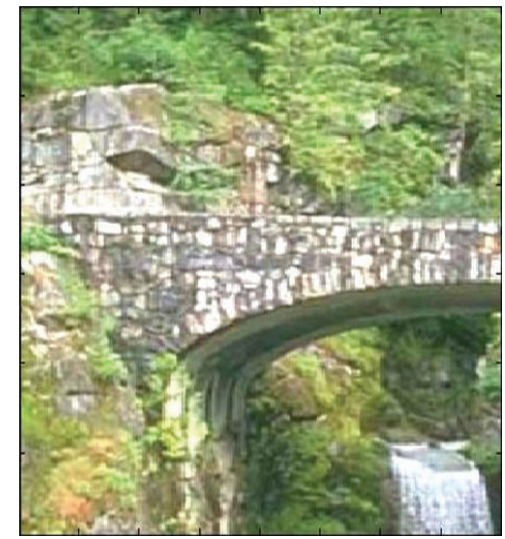

(i)

FIGURE 5: Typical results obtained from the compared algorithms of the natural images: (a) original image, (b) CIEL ${ }^{*} a^{*} b^{*}$ color model, (c) noisy and blurred image, (d) WTHE, (e) fuzzy, (f) GA, (g) PSO method, (h) DE method, and (i) proposed method.

Habitat with maximum $f$ value is said to have high HSI and vice versa.

Step 5 (identification of elite habitats). In $\mathrm{BBO}$ the best habitats in each iteration are preserved from modifications caused by migration and mutation. Thus an elitism parameter is used to provide a memory for the algorithm. Based on the HSI values of each habitat, which refers to the edge intensity variation of the image through iteration, a set of " $p$ " elite habitats are preserved. Habitats with the best (i.e., maximum) pixel value are chosen as elite habitats in each iteration.
Step 6 (blended migration). (6.1) Calculate the immigration rate $\lambda_{i}$ and emigration rate $\mu_{i}$ for each habitat using (11) and (12).

(6.2) Calculate $\lambda$ scale for each habitat using (16):

$$
\begin{aligned}
\lambda_{\text {scale }}= & \lambda_{\text {lower }}+\left(\lambda_{\text {upper }}-\lambda_{\text {lower }}\right) \\
& * \frac{(\lambda(k)-\lambda \min )}{(\lambda \max -\lambda \min )},
\end{aligned}
$$




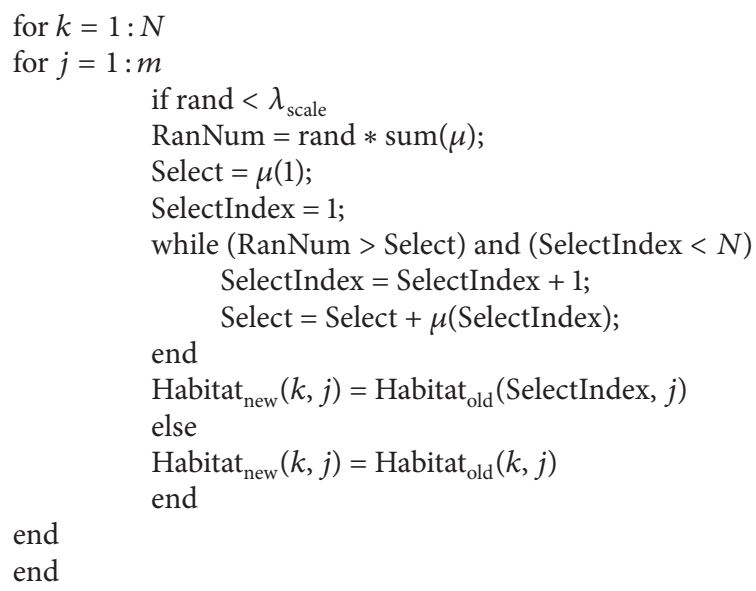

Algorithm 1

where $\lambda_{\text {lower }} / \lambda_{\text {upper }}$ are user defined parameters, $\lambda$ min is the minimum value of the lambda, and $\lambda$ max is the maximum value of the lambda.

(6.3) The migration operation for each nonelite habitat is performed as shown in Algorithm 1.

Step 7 (mutation). (7.1) Update the species count probability for each habitat using (15).

(7.2) Elitism is implemented by setting species count probability to zero for $p$ elite habitats

(7.2) Mutation process for each nonelite habitat is carried out as shown in Algorithm 2.

Step 8 (termination criterion). Check for the termination criteria. If maximum generation is reached, stop execution; otherwise go to Step 3.

\section{Experimental Results and Discussions}

5.1. Experimental Setup. The optimization problem considered in this paper is to solve the image enhancement problem using BBO-BMO approach. Our goal is to maximize the objective function in order to enhance the contrast by maximizing the number of pixels in the edges, increase the overall edges intensity, and increase the entropy measure [47]. Figure 3 shows the texture and edge intensity of the natural images. The performance of IE using the proposed method is validated by applying it to various natural images. The results obtained were compared with WTHE [7], fuzzy algorithm [18], GA [17], PSO [27], and DE [22].

5.2. Qualitative Assessment on Natural Images. Image enhancement can be easily extended to color natural images. The most obvious way to extend the grayscale IE to color images is to apply the method to luminance components only and to preserve the chrominance components. Few examples of natural images were shown in Figures 4 and 5.
Figure 4(b) is the $\operatorname{CIEL}^{*} a^{*} b^{*}$ image and Figure 4(c) is the noisy image. This image has poor contrast quality. Figure $4(\mathrm{~d})$ is the WTHE image; this image has nonuniform illumination. This becomes more apparent as it stretches the histogram to increase the contrast by darkening the pixels in the lower range and brightening the pixel in the higher range. Figure 4(e) is the fuzzy image; this image preserves the brightness and keeps the details by increasing the contrast. It has an unnatural look in Figure 4(f) GA performs better than Fuzzy it does not remove this effect completely. In Figures 4(g) and 4(h) PSO image and DE image performs better than GA but it does not preserve the edge. The proposed algorithm on the other hand does not darken the images as much as WTHE, and it preserves the bright regions, enhances the edge intensity and entropy, and as a result produces a better contrast enhance result by preserving the edges.

In Figure 5(d) WTHE results in loss of details. Figures 5(e) and 5(f) fuzzy and GA result a better than WTHE. It still has an unnatural look. In Figures 5(g) and 5(h) PSO and DE show results better than GA; however, the result in images is not as pleasing as the one obtained with the proposed method. Some portions are still brighter and edge intensity is not enhanced. The proposed method in Figure 5(i) gives a more natural looking image by preserving the brightness and enhancing the edge intensity.

5.3. Quantitative Assessment. Quality assessment of image enhancement is not an easy task; it is desirable to have an objective assessment approach to compare image enhancement techniques. There are some metrics that approximate an average contrast in the image based on entropy or other measures. If these metrics are used, $\mathrm{BBO}-\mathrm{BMO}$ can achieve the best performance even though it may not produce the visually pleasing image and possibly may produce an unrealistic look. Hence, we will use the following quantitative measures: peak signal noise ratio (PSNR), the discrete entropy $(H)$, and the fitness criteria [48-50].

The performance of image enhancement technique can be measured by comparing PSNR value. The PSNR value for enhancing the image using the proposed algorithm is compared with other traditional techniques and observations are shown in Table 1 which shows the PSNR values compared with traditional IE techniques and the proposed method. Table 2 shows the PSNR values compared with traditional IE techniques and the proposed method for different iterations.

The discrete entropy is used to measure the content of an image, where a higher value indicates an image with richer details. The entropy approximates an average contrast in the image by dividing image into nonoverlapping blocks, finding a measure based on minimum and maximum intensity values in each block and averaging them. It is computed pixelby-pixel by adding up the squared differences of all the pixels and dividing by the total pixel count. Table 3 shows the comparison of entropy values with the traditional IE techniques.

The performance can be measured by comparing the fitness value of some optimization algorithms with that of the proposed algorithm. Table 4 shows the fitness comparison of 


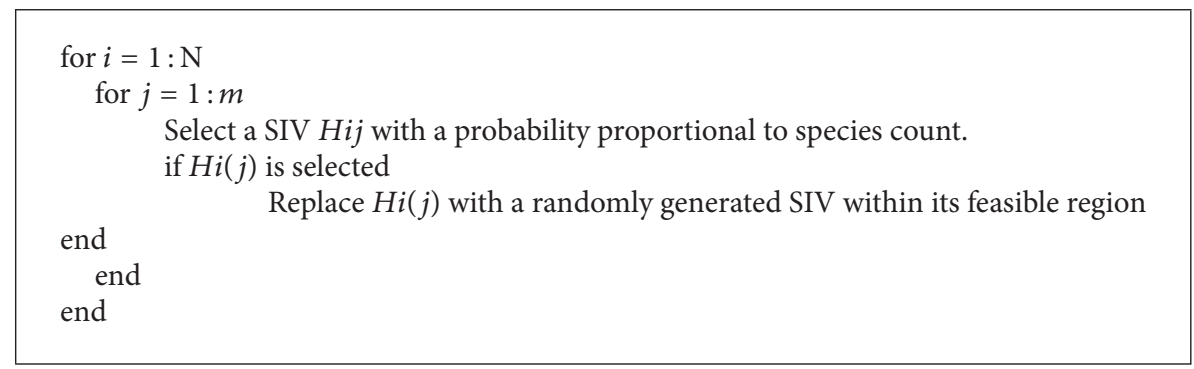

Algorithm 2

TABLE 1: PSNR values compared with traditional IE techniques

\begin{tabular}{lcccc}
\hline Images & Fuzzy & GA & PSO & BBO-BMO \\
\hline Image 1 & 44.496 & 45.745 & 47.506 & 50.516 \\
Image 2 & 44.636 & 45.605 & 46.854 & 48.615 \\
Image 3 & 45.032 & 46.001 & 47.25 & 49.011 \\
Image 4 & 46.001 & 47.25 & 49.011 & 50.22 \\
\hline
\end{tabular}

TABLE 2: PSNR values compared with traditional IE techniques for different iterations.

\begin{tabular}{lcccc}
\hline Iterations & Fuzzy & PSO & GA & BBO-BMO \\
\hline 5 & 44.96 & 45.45 & 47.806 & 50.316 \\
50 & 44.36 & 45.05 & 46.654 & 48.215 \\
100 & 45.532 & 46.301 & 47.425 & 49.611 \\
200 & 44.496 & 45.745 & 47.506 & 49.516 \\
300 & 44.636 & 45.605 & 46.854 & 48.615 \\
400 & 45.032 & 46.001 & 47.25 & 49.011 \\
500 & 46.001 & 47.25 & 49.011 & 50.22 \\
\hline
\end{tabular}

TABle 3: Discrete entropy $H$ compared with traditional IE techniques.

\begin{tabular}{lcccccc}
\hline Image & WTHE & Fuzzy & GA & DE & PSO & BBO-BMO \\
\hline Image 1 & 7.315 & 7.215 & 7.352 & 7.52 & 7.492 & 6.629 \\
Image 2 & 7.808 & 7.774 & 7.798 & 7.478 & 7.497 & 7.774 \\
Image 3 & 7.565 & 7.678 & 7.565 & 7.555 & 7.549 & 7.603 \\
Image 4 & 7.438 & 7.558 & 7.543 & 7.552 & 7.604 & 6.881 \\
\hline
\end{tabular}

TABLE 4: Fitness values $F(Z)$ for the optimization algorithms.

\begin{tabular}{lccccc}
\hline Image & Fuzzy & GA & DE & PSO & BBO-BMO \\
\hline Image 1 & 253.699 & 258.627 & 259.119 & 260.209 & 265.711 \\
Image 2 & 257.223 & 263.436 & 265.938 & 269.198 & 275.413 \\
Image 3 & 277.975 & 283.412 & 285.236 & 286.546 & 290.254 \\
Image 4 & 261.333 & 271.864 & 272.155 & 274.345 & 281.567 \\
\hline
\end{tabular}

the proposed algorithm based IE technique with the Fuzzy, GA, DE and PSO.

The parameters of these algorithms are set as described in their own paper. Since the compared algorithms except WTHE are stochastic and population based methods, 30 independent runs were made for each of the methods to show the efficiency of these algorithms. We implemented the BBOBMO algorithm in Matlab with a $1.73 \mathrm{GHz}$ Intel Pentium Dual-Core processor with $2 \mathrm{~GB}$ of random access memory.

\section{Conclusion}

In this paper a new $\mathrm{BBO}-\mathrm{BMO}$ based color image enhancement technique is proposed. This algorithm is employed with few natural images and the results obtained are compared with other population based methods. By maximizing the fitness criterion and adopting the parameters the algorithm enhances the contrast and detail of the image. The proposed method is applicable to a wide variety of natural images. From many decades, image enhancement is implemented using techniques like PSO and GA. BBO-BMO is uniquely a biogeography technique used for implementing image enhancement which provides more accurate enhanced images as compared to other evolutionary algorithms. $\mathrm{BBO}-\mathrm{BMO}$ is a population based optimization algorithm and it does not involve reproduction or the generation of "children." The simulation results show the effectiveness of the algorithm in terms of quality assessment and computation efficiency with the other IE algorithms. The obtained results are visually pleasing, artifact free, and natural looking. Comparative experimental results on real-world natural images have demonstrated the efficiency and effectiveness of our proposed method.

\section{Conflict of Interests}

The authors declare that there is no conflict of interests regarding the publication of this paper.

\section{References}

[1] Rafael, C. Gonzalez, and E. Richard Woods, Digital Image Processing, Prentice Hall, 2nd edition, 2009.

[2] A. K. Jain, Fundamentals of Digital Image Processing, Prentice Hall, Englewood Cliffs, NJ, USA, 1991.

[3] R. C. Gonzalez and B. A. Fittes, "Gray-level transformations for interactive image enhancement," Mechanism and Machine Theory, vol. 12, no. 1, pp. 111-122, 1977.

[4] V. Buzuloiu, M. Ciuc, R. M. Rangayyan, and C. Vertan, "Adaptive-neighborhood histogram equalization of color images," International Journal of Electronic Imaging, vol. 10, no. 2, pp. 445-459, 2001. 
[5] C.-C. Sun, S.-J. Ruan, M.-C. Shie, and T. W. Pai, "Dynamic contrast enhancement based on histogram specification," IEEE Transactions on Consumer Electronics, vol. 51, no. 4, pp. 13001305, 2005.

[6] A. Tarik, D. Salih, and A. Yucel, "A histogram modification framework and its application for image contrast enhancement," IEEE Transactions on Image Processing, vol. 18, no. 9, pp. 1921-1935, 2009.

[7] Q. Wang and R. K. Ward, "Fast image/video contrast enhancement based on weighted thresholded histogram equalization," IEEE Transactions on Consumer Electronics, vol. 53, no. 2, pp. 757-764, 2007.

[8] R. Namoto Matsubayasi, T. Fujii, K. Yasumori, T. Muranaka, and S. Momosaki, "Apparent diffusion coefficient in invasive ductal breast carcinoma: correlation with detailed histologic features and the enhancement ratio on dynamic contrast-enhanced MR images," Journal of Oncology, vol. 2010, Article ID 821048, 6 pages, 2010.

[9] A. Paul, J. Yung-Chuan, W. Jhing-Fa, and Y. Jar-Ferr, "Parallel reconfigurable computing based mapping algorithm for motion estimation in advance video coding," ACM Transaction on Embedded Computing Systems, vol. 11, no. S2, article 40, 2012.

[10] G. Janssnes, L. Jacques, J. Ordan Xivry, X. Geets, and B. Macq, "Diffeomorphic registration of images with variable contrast enhancement," International Journal of Biomedical Imaging, vol. 2011, Article ID 891585, 16 pages, 2011.

[11] B. Tang, G. Sapiro, and V. Caselles, "Color image enhancement via chromaticity diffusion," IEEE Transactions on Image Processing, vol. 10, no. 5, pp. 701-707, 2001.

[12] T. Back, D. Fogel, and Z. Michalewicz, Handbook of Evolutionary Computation, Oxford University Press, Oxford, UK, 1997.

[13] C. Munteanu and A. Rosa, "Gray-scale image enhancement as an automatic process driven by evolution," IEEE Transactions on Systems, Man, and Cybernetics B, vol. 34, no. 2, pp. 1292-1298, 2004.

[14] P. Knoll and S. Mirzaei, "Validation of a parallel genetic algorithm for image reconstruction from projections," Journal of Parallel and Distributed Computing, vol. 63, no. 3, pp. 356359, 2003.

[15] S. M. Guo, C. S. Lee, and C. Y. Hsu, "An intelligent image agent based on soft-computing techniques for color image processing," Expert Systems with Applications, vol. 28, no. 3, pp. 483-494, 2005.

[16] C.-C. Lai and D.-C. Tseng, "An optimal $L$-filter for reducing blocking artifacts using genetic algorithms," Signal Processing, vol. 81, no. 7, pp. 1525-1535, 2001.

[17] M. Shyu and J. Leon, "A genetic algorithm approach to color image enhancement," Pattern Recognition, vol. 31, no. 7, pp. 871$880,1998$.

[18] H. D. Cheng and H. J. Xu, "A novel fuzzy logic approach to contrast enhancement," Pattern Recognition, vol. 33, no. 5, pp. 809-819, 2000.

[19] Y. Shkvarko, H. Perez-Meana, and A. Castillo-Atoche, "Enhanced radar imaging in uncertain environment: a descriptive experiment design regularization approach," International Journal of Navigation and Observation, vol. 2008, Article ID 810816, 11 pages, 2008.

[20] S. Palanikumar, M. Sasikumar, and J. Rajeesh, "Entropy optimized palmprint enhancement using genetic algorithm and histogram equalization," International Journal of Genetic Engineering, vol. 2, pp. 12-18, 2012.
[21] S. Hashemi, S. Kiani, N. Noroozi, and M. E. Moghaddam, "An image contrast enhancement method based on genetic algorithm," Pattern Recognition Letters, vol. 31, no. 13, pp. 18161824, 2010.

[22] L. dos Santos Coelho, J. Guilherme Sauer, and M. Rudek, "Differential evolution optimization combined with chaotic sequences for image contrast enhancement," Chaos, Solutions and Fractals, vol. 42, pp. 522-529, 2009.

[23] N. M. Kwok, Q. P. Ha, D. Liu, and G. Fang, "Contrast enhancement and intensity preservation for Gray-level images using multi objective particle swarm optimization," IEEE Transactions on Automation Science and Engineering, vol. 6, no. 1, pp. 145-156, 2009.

[24] P. Shanmugavadivu and K. Balasubramanian, "Particle swarm optimized multi-objective histogram equalization for image enhancement," Optics \& Laser Technology, vol. 57, pp. 243-251, 2014.

[25] S. Xiaoping, F. Wei, S. Qing, and H. Xjulan, "An image enhancement method using the quantum-behaved particle swarm optimization with an adaptive strategy," Mathematical Problems in Engineering, vol. 2013, Article ID 824787, 14 pages, 2013.

[26] K. Gaurav and H. Bansal, "Particle Swarm Optimization (PSO) technique for image enhancement," International Journal of Electronics \& Communication Technology, vol. 4, no. 3, pp. 117119, 2013.

[27] A. Gorai and A. Ghosh, "Hue-preserving color image enhancement using particle swarm optimization," in Proceedings of the IEEE Transactions on Evolutionary Computation, pp. 563-568, 2011.

[28] S. Agrawal and R. Panda, "An efficient algorithm for Gray level image enhancement using Cuckoo search," in Swarm, Evolutionary, and Memetic Computing, vol. 7677 of Lecture Notes in Computer Science, pp. 82-89, 2012.

[29] M. Hanmandlu, D. Jha, and R. Sharma, "Color image enhancement by fuzzy intensification," in Proceedings of the International Conference on Pattern Recognition, 2000.

[30] A. Toet, "A hierarchical morphological image decomposition," Pattern Recognition Letters, vol. 11, no. 4, pp. 267-274, 1990.

[31] W. Shibin, Y. Shaode, Y. Yuhan, and X. Yaoqin, "Feature and contrast enhancement of mammographic image based on multiscale analysis and morphology," Computational and Mathematical Methods in Medicine, vol. 2013, Article ID 716948, 8 pages, 2013.

[32] R. N. Strickland, C. S. Kim, and W. F. McDonnell, "Digital color image enhancement based on thesaturation component," Optical Engineering, vol. 26, pp. 609-616, 1987.

[33] I. M. Bockstein, "Color equalization method and its application to color image processing," Journal of the Optical Society of America A, vol. 3, no. 5, pp. 735-737, 1986.

[34] S. Lee, H. Kwon, H. Han, G. Lee, and B. Kang, "A spacevariant luminance map based Color image enhancement," IEEE Transactions on Consumer Electronics, vol. 56, no. 4, pp. $2636-$ 2643, 2010.

[35] S. K. Naik and C. A. Murthy, "Hue-preserving color image enhancement without gamut problem," IEEE Transactions on Image Processing, vol. 12, no. 12, pp. 1591-1598, 2003.

[36] T. Adlin Sharo and K. Raimond, "Enhancing degraded color images using Fuzzy logic and artificial Bee colony," International Journal of Computational Engineering Research, vol. 3, no. 3, pp. 356-361, 2013. 
[37] O. Prakash Verma, P. Kumar, M. Hanmandlu, and S. Chhabra, "High dynamic range optimalfuzzy color image enhancement using Artificial Ant Colony System," Applied Soft Computing, vol. 12, pp. 394-404, 2011.

[38] D. Simon, "Biogeography-based optimization," IEEE Transactions on Evolutionary Computation, vol. 12, no. 6, pp. 702-713, 2008.

[39] N. Singh, M. Kaur, and K. V. P. Singh, "Parameter optimization in image enhancement using PSO," American Journal of Engineering Research, vol. 2, no. 5, pp. 84-90, 2013.

[40] M. Braik, A. Sheta, and A. Ayesh, "Particle swarm optimisation enhancement approach for improving image quality," International Journal of Innovative Computing and Applications, vol. 1, no. 2, pp. 138-145, 2007.

[41] C. Munteaunu and A. Rosa, "Towards automatic image enhancement using genetic algorithms," in Proceedingsof the Congress on Evolutionary Computation, vol. 2, pp. 1535-1542, San Diego, Calif, USA.

[42] Y. Sun, P. Wu, G. W. Wei, and G. Wang, "Evolution-operatorbased single-step method for image processing," International Journal of Biomedical Imaging, vol. 2006, Article ID 83847, 27 pages, 2006.

[43] H. Ma and D. Simon, "Blended biogeography-based optimization for constrained optimization," Engineering Applications of Artificial Intelligence, vol. 24, no. 6, pp. 517-525, 2010.

[44] F. Russo, "An image enhancement technique combining sharpening and noise reduction," IEEE Transactions, vol. 51, no. 4, pp. 824-828, 2002.

[45] B. Zhang and J. P. Allebach, "Adaptive bilateral filter for sharpness enhancement and noise removal," IEEE Transactions on Image Processing, vol. 17, no. 5, pp. 664-678, 2008.

[46] A. Beghadadi and A. L. Negrate, "Contrast enhancement technique based on local detection of edges," Computer Vision, Graphics, and Image Processing, vol. 46, no. 3, pp. 162-174, 1989.

[47] C. Kuo-Liang, Y. Wei-Jen, and Y. Wen-Ming, "Efficient edgepreserving algorithm for colorcontrast enhancement with application to color image segmentation," Journal of Visual Communication and Image Representation, vol. 19, no. 5, pp. 299-310, 2008.

[48] S. D. Chen and A. Ramli, "Minimum mean brightness error bihistogram equalization in contrastenhancement," IEEE Transactions on Consumer Electronics, vol. 49, no. 4, pp. 1310-1319, 2003.

[49] S. S. Agaian, K. Panetta, and A. M. Grigoryan, "Transformbased image enhancement algorithms with performance measure," IEEE Transactions on Image Processing, vol. 10, no. 3, pp. 367-382, 2001.

[50] L. Zhang and X. Mou, "FSIM: a feature similarity index for image quality assessment," IEEE Transactions on Image Processing, vol. 20, no. 8, pp. 2378-2386, 2011. 


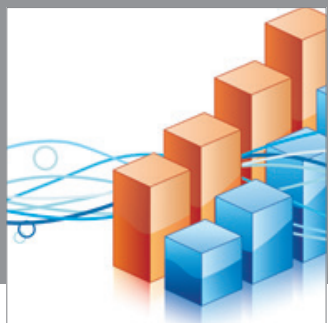

Advances in

Operations Research

mansans

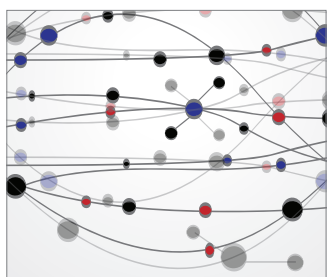

The Scientific World Journal
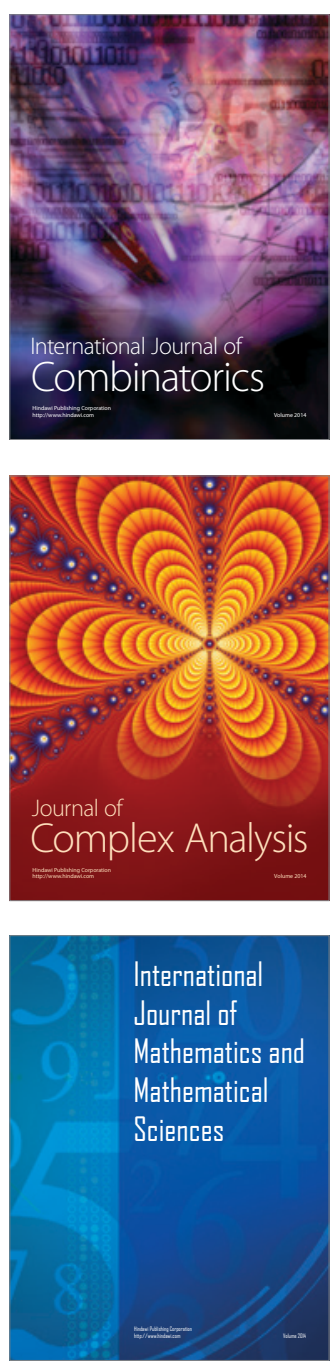
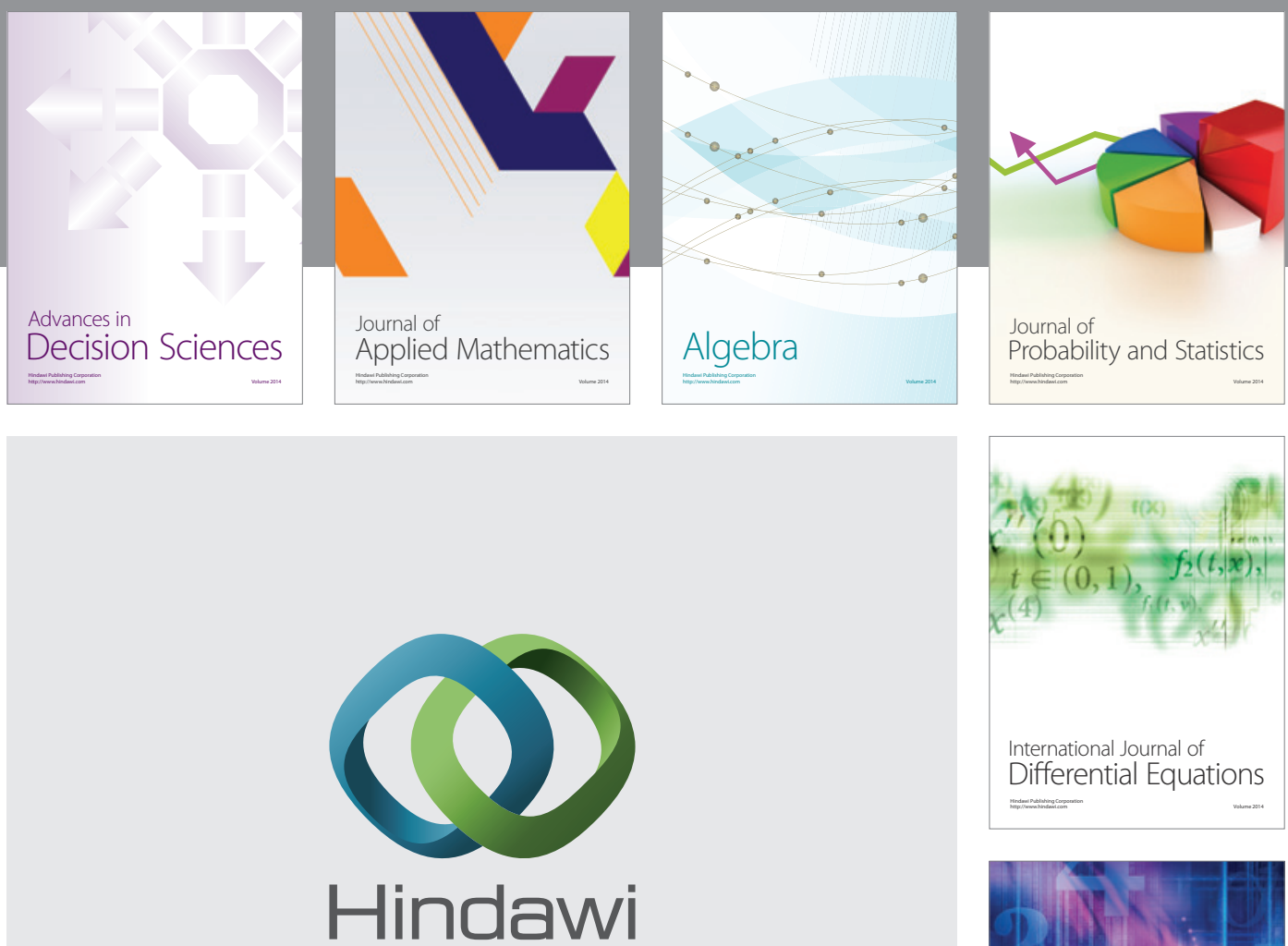

Submit your manuscripts at http://www.hindawi.com
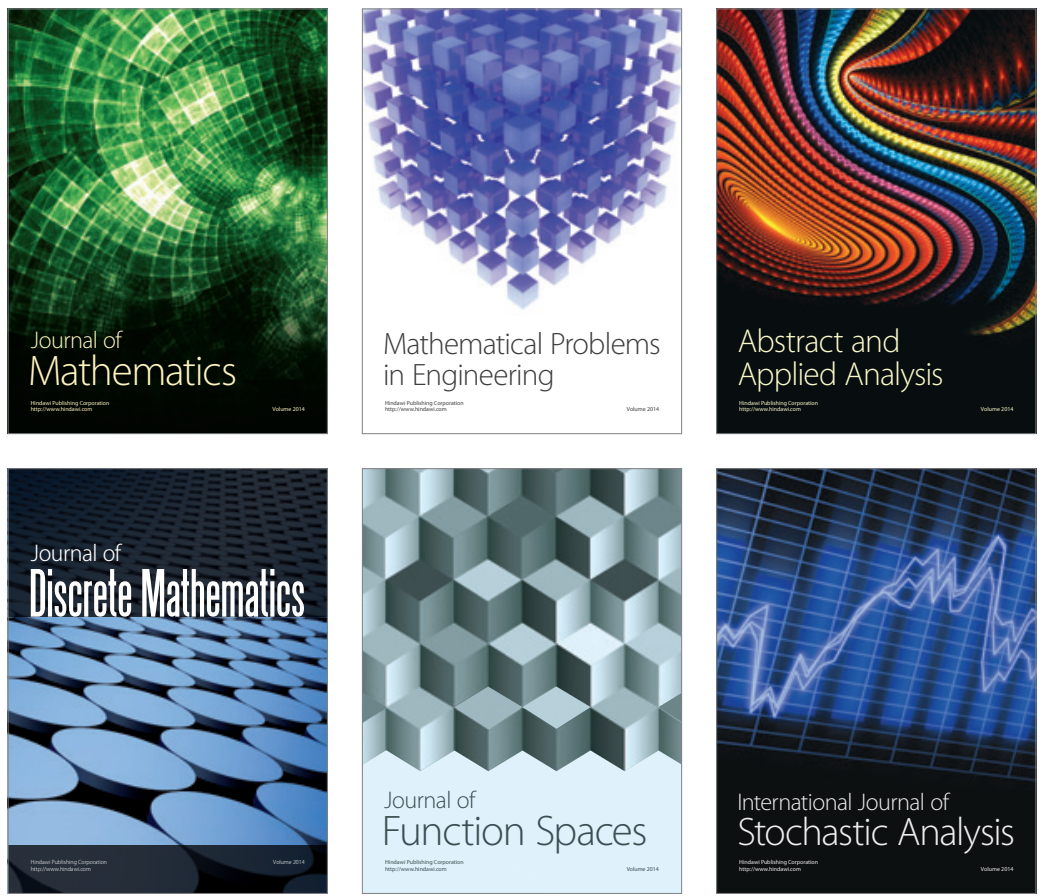

Journal of

Function Spaces

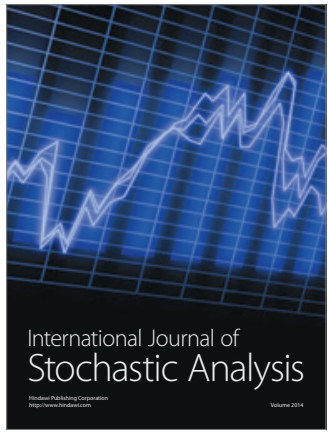

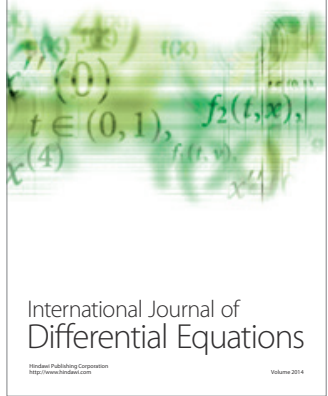
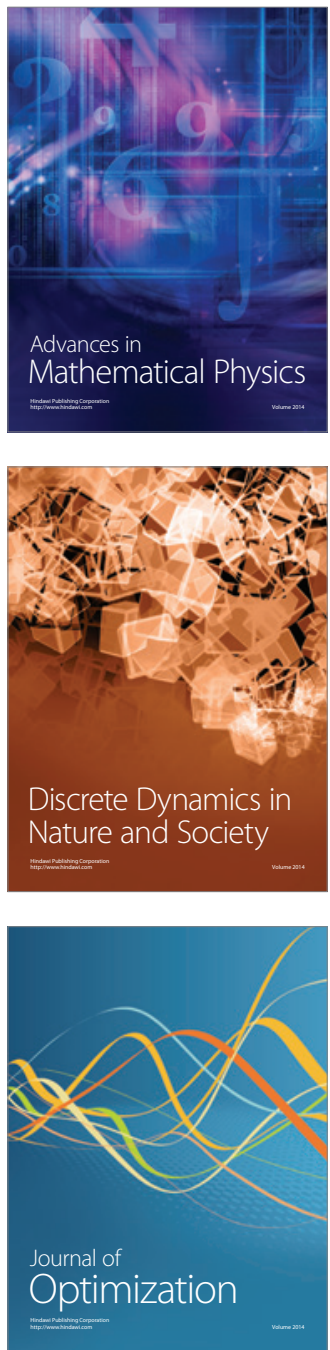